\title{
Ácaros de penas e carrapatos (Acari) associados a Turdus albicollis Vieillot (Aves, Muscicapidae) em uma área de Mata Atlântica da llha Grande, Rio de Janeiro, Brasil
}

\author{
Alline Storni ${ }^{1}$, Maria A. S. Alves ${ }^{1} \&$ Michel P. Valim ${ }^{2}$ \\ ${ }^{1}$ Departamento de Ecologia, Universidade do Estado do Rio de Janeiro. Rua São Francisco Xavier 524, Maracanã, \\ 20550-011 Rio de Janeiro, Rio de Janeiro,Brasil.E-mail: masa@uerj.br \\ ${ }^{2}$ Departamento de Entomologia, Fundação Oswaldo Cruz, Instituto Oswaldo Cruz. Avenida Brasil 4365, Manguinhos, \\ 21045-900 Rio de Janeiro, Rio de Janeiro, Brasil.
}

\begin{abstract}
Feather mites and ticks (Acari) associated to Turdus albicollis Vieillot (Aves, Muscicapidae) in an area of Atlantic Forest at Ilha Grande, Rio de Janeiro, Brasil. Parasitism is an important mechanism affecting populations and communities. The purpose of this study was to investigate the ectoparasites fauna living on the body of the white-necked trush, Turdus albicollis Vieillot, 1818, and to evaluate if the host body mass is affected by these parasites. Turdus albicollis were monthly captured at Ilha Grande, Rio de Janeiro, from July 1999 to June 2000 in an area of Atlantic Forest. The birds were individualy marked, weighed and carefully checked to record and quantify the presence of ectoparasites. Parasite abundance and location on the bird's body were recorded. In 54 individuals of $T$. albicollis sampled, two ectoparasite species were found. The prevalence of the feather mite Pterodectes turdinus Berla, 1959, and the tick Amblyomma longirostre Koch, 1844 was $72,2 \%$ and $27,8 \%$, respectively. The monthly abundance of $P$. turdinus was related to the dry and wet months, and it was significantly higher in the dry months than in the wet months. There was no significant relationship between hosts body mass ( $\mathrm{g}$ ) and total abundance of feather mites and ticks.

KEY WORDS. Amblyomma longirostre, ectoparasitism, host, Pterodectes turdinus.
\end{abstract}

RESUMO. O parasitismo é um importante mecanismo que afeta populações e comunidades. O objetivo deste estudo foi investigar a fauna de ectoparasitos que habita o corpo do sabiá-de-coleira, Turdus albicollis Vieillot, 1818 e avaliar se a massa corporal do hospedeiro é afetada por estes parasitos. Os indivíduos de $T$. albicollis foram mensalmente capturados na llha Grande, Rio de Janeiro, no período de julho de 1999 a junho de 2000, em uma área de Floresta Atlântica. As aves foram individualmente marcadas, pesadas e examinadas para registrar e quantificar a presença de ectoparasitos. A abundância e a localização dos parasitos no corpo do hospedeiro foram registradas. Em 54 indivíduos de T. albicollis amostrados, foram encontrados duas espécies de ectoparasitos. A prevalência de ácaros de penas, Pterodectes turdinus Berla, 1959, foi de $72,2 \%$ enquanto que a de carrapatos, Amblyomma longirostre Koch, 1844 , foi de $27,8 \%$. A abundância mensal de $P$. turdinus foi significativamente relacionada com os meses do ano, sendo maior nos meses com menor freqüência de chuva. Não houve relação estatisticamente significativa entre a massa corporal do hospedeiro (g) e a abundância total de ácaros de penas e carrapatos.

PALAVRAS CHAVE. Amblyomma Iongirostre, ectoparasitismo, hospedeiro, Pterodectes turdinus.

O parasitismo, assim como a predação e a competição, constitui uma importante força seletiva em populações por reduzir a energia destinada a processos fisiológicos de seus hospedeiros (Loye \& CARrol 1995, Schall1983, Sorci et al. 1996). Os ectoparasitos afetam as aves diretamente, alimentando-se delas e indiretamente, servindo como vetores de protozoários, bactérias e vírus. Os ectoparasitos de aves, que incluem uma variedade de insetos (percevejos, pulgas e piolhos), de carrapatos e de ácaros que infestam penas, pele, passagens respiratórias e ninhos e podem deixá-las susceptíveis a infecções secundárias (PHILıPs 1990) e podem induzir anemia (LeHMAN 1993). Outros efeitos que afetam a aptidão e o sucesso reprodutivo são o atraso na postura dos ovos (Moller 1990); a diminuição no tamanho da ninhada (Moss \& Camin 1970, Lope \& MolLer 1993), causando acentuada redução da sobrevivência dos ninhegos parasitados em até 50\% (Brown \& Brown 1986), a diminuição da massa corporal dos ninhegos (Moss \& CAmin 1970, Brown \& Brown 1986, Moller 1990, Merino \& Potti 1995, Alves 1997) e de adultos (THOMPson et al. 1997).

O custo do parasitismo pode ser elevado, levando as aves muitas vezes, a construírem novos ninhos a cada estação reprodutiva, em vez de reaproveitarem ninhos construídos em esta- 
ções reprodutivas anteriores, que de maneira geral estão infestados por ectoparasitos, como por exemplo ocorre com as andorinhas das espécies Hirundo pyrrhonota (BRown \& Brown 1986) e Riparia riparia (Alves 1997).

Ectoparasitos estão sujeitos a alterações do ambiente e também a mudanças internas do corpo do hospedeiro (BEGON et al. 1990). Fatores ambientais, tais como: temperatura e pluviosidade, também têm sido mencionados para explicar diferenças sazonais na intensidade, na abundância e na prevalência parasitárias (LinARDi et al. 1985, DAVIDSON et al. 1994).

Embora diversos trabalhos apontem os ácaros de penas como parasitos (СHOE \& Kiм 1987, Thompson et al. 1997, HaRPer 1999), recentemente alguns autores têm colocado os ácaros de penas como organismos simbiontes (Blanco et al. 2001, BLANCO \& Frias 2001), vivendo na superfície das penas de vôo do hospedeiro e possivelmente alimentando-se do óleo produzido pelas glândulas nas penas e organismos que as estas penas se aderem. A partes bucais destes ácaros indicam que estes se limitam a ingerir líquidos e pequenos materiais sólidos contidos nas penas (KRANTZ 1971), mostrando assim que estes não causam nenhum efeito negativo aparente na plumagem das aves. Entretanto, apesar da importância do tema, pouco se conhece sobre o assunto na fauna neotropical.

O objetivo deste estudo foi conhecer a fauna de ectoparasitos associados a ave da espécie Turdus albicollis, em uma área de Mata Atlântica pouco perturbada antropicamente e analisar os efeitos da infestação por ectoparasitos na massa corporal de T. albicollis. O trabalho também visou analisar os microhábitats preferenciais e a abundância total de cada espécie de ectoparasito no corpo do hospedeiro, além de avaliar o efeito da pluviosidade na intensidade de infestação de ectoparasitos em T. albicollis.

\section{MATERIAL E MÉTODOS}

A área de estudo situa-se na Ilha Grande $\left(23^{\circ} 11^{\prime}\right.$, $\left.44^{\circ} 12^{\prime} \mathrm{W}\right)$, na região da Baía da Ilha Grande, no município de Angra dos Reis, Rio de Janeiro. O estudo foi desenvolvido em uma área de Mata Atlântica pouco perturbada antropicamente, localizada aproximadamente a $240 \mathrm{~m}$ de altitude, e consideravelmente bem preservada. Nesta área foi marcada uma grade de 5,0 ha, com 16 trilhas paralelas distando $20 \mathrm{~m}$ entre si, ao longo das quais as redes foram colocadas. O clima é quente e úmido, com uma temperatura média anual de $23^{\circ} \mathrm{C}$. A pluviosidade anual na área é de aproximadamente $2200 \mathrm{~mm}$ (dados obtidos através do Sistema de Metereologia da Central Nuclear Almirante Álvaro Alberto - CNAAA; Gerência de Metereologia da Eletrobrás Termonuclear S.A. - Eletronuclear).

As aves foram capturadas entre julho de 1999 e junho de 2000, perfazendo um total de dois dias de subseqüentes trabalho por mês na área amostrada. Foram utilizadas 10 redes ornitológicas (12,0 m x 2,5 m e malha $36 \mathrm{~mm}$ ) que permaneceram abertas durante um período de sete horas por dia (a partir de 06:30 h), totalizando 168 horas/rede de amostragem ao fi- nal do estudo. Estas redes foram revisadas a intervalos de tempo regulares, nunca superiores a uma hora para vistoriar eventuais capturas de T. albicollis.

Após a captura, as aves foram acondicionadas individualmente em sacos limpos feitos com tecido de algodão, de forma a evitar que os ectoparasitos desprendidos do corpo do hospedeiro e que eventualmente tivessem permanecido no interior do saco se transferissem para outra ave. Os parasitos foram amostrados sempre exclusivamente na primeira captura de cada ave para manter a independência das amostras.

Depois de recolhidos os ectoparasitos, as aves foram individualmente marcadas com anilhas metálicas (fornecidas pelo CEMAVE/IBAMA), pesadas com dinamômetros Pesola (precisão de $0,5 \mathrm{~g}$ ) e com balança de precisão do tipo Acculab V-200 (precisão de 0,01 g).

Para avaliar a presença ou não de ácaros de pena foi feita uma inspeção no corpo da ave, com auxílio de uma lupa de mão que continha uma lâmpada e uma área circular de aproximação que sempre foi colocada a uma distância aproximada de $10 \mathrm{~cm}$, das rêmiges e das retrizes. De cada uma das penas das asas foi realizada a contagem de maneira padronizada para todos os indivíduos. Os carrapatos foram amostrados soprando-se as penas até que a pele pudesse ser visualizada. Este método se mostrou eficaz e foi padronizado para todas as aves amostradas.

Foram calculadas as prevalências para ácaros de penas e carrapatos segundo Bush et al. (1997), que define prevalência como o número de hospedeiros infestados com determinado parasito dividido pelo número total de hospedeiros examinados, com o valor dado em porcentagem.

Foi registrada a ocorrência dos ectoparasitos em cada uma das regiões do corpo em que foram encontrados. Este procedimento visou a identificação dos microhábitats preferenciais e a abundância total para cada espécie de ectoparasito no corpo do hospedeiro.

Foram coletadas algumas penas infestadas com ácaros e os carrapatos foram removidos com o auxílio de uma pinça. Todas as amostras foram conservadas em frascos individuais com álcool 70\% devidamente etiquetados para posterior identificação.

No laboratório, o conteúdo dos frascos foi examinado sob microscópio estereoscópico e separado individualmente para a montagem das lâminas. Os espécimes de ácaros de pena foram clarificados com lactofenol, montados em lâminas com meio de Hoyer, e examinados com o auxílio de microscópio para identificação, seguindo metodologia proposta por FACCINI et al. (1976). A identificação foi feita com chave dicotômica de Gaud \& Atyeo (1996). Os carrapatos foram identificados com auxílio de microscópio estereoscópico e de chave dicotômica de Keirans \& Durden (1998) para ninfas e de Amorim \& SerraFReIRE (1999) para larvas.

Para avaliar a influência de ectoparasitos (abundância total) na massa corporal do hospedeiro, foi realizada a análise de correlação de Spearman, uma vez que a abundância total de 
ectoparasitos não teve distribuição normal quando testada no Programa Estatístico Systat for Windows pela análise de Kurtosis e Skewness (ZAR 1984).

As abundâncias mensais de cada espécie de ectoparasito foram calculadas para os meses com maior e menor freqüência de chuva e comparadas pelo teste $U$ de Mann-Whithney (ZAR 1984). Foram considerados os meses secos aqueles com uma precipitação inferior a $120 \mathrm{~mm}$, enquanto os meses chuvosos foram aqueles que apresentaram uma precipitação igual ou superior a este valor. Assim os meses de julho a setembro de 1999 e os meses de maio e junho de 2000 foram considerados como meses secos, enquanto os meses de outubro a dezembro de 1999 e de janeiro a abril de 2000 foram tratados como chuvosos.

\section{RESULTADOS}

Foram capturados 54 indivíduos de T. albicollis, dentre os quais 39 estavam infestados com 7338 Pterodectes turdinus Berla, 1959 (Acari: Proctophyllodidae), com uma prevalência de 72,2\%. Em relação aos carrapatos, todos indivíduos de Amblyomma longirostre $(\mathrm{n}=41)$, foram encontrados em 15 indivíduos de $T$. albicollis, perfazendo uma prevalência de $27,8 \%$ das aves analisadas. Dos indivíduos de T. albicollis que continham carrapatos, $20 \%$ também possuíam simultaneamente ácaros de pena. Os carrapatos encontrados associados a T. albicollis estavam todos em estádios iniciais de vida (larvas e ninfas).

Em 72,2\% dos indivíduos de T. albicollis infestados com $P$. turdinus, os ácaros estavam parasitando suas rêmiges, não tendo sido registrado a presença destes ácaros em penas de contorno ou nas retrizes. Dos 41 carrapatos encontrados associados a T. albicollis, $82,9 \%$ foram encontrados na parte superior dos olhos, $14,6 \%$ no pescoço e $2,4 \%$ próximos ao bico.

A abundância mensal de ácaros de pena em T. albicollis diferiu significativamente entre os meses secos e chuvosos (Mann-Whitney, $U=30, p=0,032$ ), sendo maior nos meses secos. Entretanto, não houve relação estatisticamente significativa entre a abundância mensal de carrapatos e os meses secos e chuvosos (Mann-Whitney, $U=18,5 \mathrm{p}=0,337$ ).

Não houve correlação entre a abundância total de $P$. turdinus com a massa corporal de T. albicollis (Correlação de Spearman $\left.r_{s}=0,010 ; N=43, p>0,05\right)$. Também não foi encontrada correlação entre a abundância total de carrapatos com a massa corporal de T. albicollis (Correlação de Spearman $\mathrm{r}_{\mathrm{s}}=$ $0,242 ; \mathrm{N}=43, \mathrm{p}>0,05)$.

\section{DISCUSSÃO}

Neste estudo foi observada uma elevada prevalência (72,22\%) por ácaros de pena do gênero Pterodectes Robin \& Mégnin, 1877, o que está de acordo com os resultados obtidos em outros estudos com aves silvestres em área de Mata Atlântica de Pernambuco (Neves et al. 2000). Marini et al. (1996) registraram $100 \%$ de ocorrência de infestação em T. albicollis por ácaros de pena e $54,6 \%$ por carrapatos.
Carrapatos do gênero Amblyomma Koch, 1844 também foram tidos como ectoparasitos freqüentes no trabalho realizado por Neves et al. (2000), apresentando uma freqüência de 100\% na comunidade estudada. No presente estudo foram registradas apenas ninfas e larvas de A. longirostre. Rojas et al. (1999) estudando aves silvestres em florestas e cerrados em Minas Gerais encontraram apenas ninfas e larvas de Amblyomma cajennense na comunidade de aves estudadas, inclusive em T. albicollis. Das 102 espécies de Amblyomma, 37 são encontradas em répteis na forma adulta e 20 são encontradas em estádios imaturos em aves (LopEs et al. 1998). Estes estudos sugerem que as aves são hospedeiras de transporte destes carrapatos, ajudando na dispersão da fase imatura (larvas e ninfas), mas não da forma adulta.

Alguns autores reportaram resultados semelhantes aos encontrados no presente trabalho ao estudarem os principais microhabitats utilizados por carrapatos em espécies de aves (MARINI et al. 1996, RoJAs et al. 1999). Estes resultados sugerem que estes locais onde se alojam os carrapatos no corpo do hospedeiro são de difícil acesso, o que torna pouco viável a remoção destes parasitos pelo hospedeiro. CHOE \& KIM (1987) comentaram que, ou as aves realmente retiram estes parasitos com o bico, ou os ectoparasitos selecionam os locais apropriados para se instalarem.

Os dados obtidos no presente estudo não permitem afirmar que a presença de ácaros de penas tenha afetado a massa corporal de T. albicollis. De maneira similar, a presença de carrapatos também, aparentemente, não afetou a massa corporal desta espécie de ave. MARINI et al. (1996), trabalhando com aves silvestres, também não encontraram uma correlação significativa entre a massa corporal dos hospedeiros e a infestação de ectoparasitos.

Os ácaros de penas alimentam-se de fragmentos das penas e de descamação da pele, assim como das secreções encontradas nas penas (GAud \& ATYEo 1996). Philips (1990) sugeriu que aves podem suportar populações de centenas de ácaros de pena sem apresentar nenhum efeito patológico, o que representaria não um parasitismo mas sim uma relação de comensalismo. GAUD \& Aтүео (1996) também sugeriram que os ácaros de pena são comensais, mantendo-se no corpo do hospedeiro para realizar seu ciclo vital. Porém, estes autores, neste mesmo trabalho, mostraram um declínio da massa corporal e na produção de ovos de galinhas, causado por uma grande infestação de ácaros de pena do gênero Megninia Berlese, 1883 (Analgidae). Entretanto, vale ressaltar que estes resultados foram decorrentes de aves criadas em condições de cativeiro. Ocasionalmente ácaros de pena são encontrados fora de seus microhábitas (GAUD \& ATYEO 1996). Mumcuoglu \& Muller (1974) encontraram dois gêneros de ácaros de pena nos pulmões de um indivíduo de Bubo bubo (Linnaeus, 1758). Eles sugeriram que o mal causado por nematóides e ácaros nos pulmões possibilitou uma possível infecção secundária que resultou em morte deste animal. GAUD (1961) notou que a infestação por ácaros de pena pode danificar as penas das aves, mas em T. albicollis na Ilha Grande nenhum dano aparente às penas das aves foi observado.

Revista Brasileira de Zoologia 22 (2): 419-423, junho 2005 
GaUd \& ATyeo (1996) confirmaram que a maioria dos ácaros de pena são encontrados nas rêmiges, porém também podem ser encontrados em retrizes e penas do corpo com menos abundância. Philips (1990) mostrou que diferentes espécies de ácaros de pena, ou diferentes estádios da mesma espécie preferem diferentes penas ou diferentes partes da mesma pena. PÉrez \& ATYeo (1984) sugeriram que a partilha de microhábitats permite que diferentes espécies de ácaros de pena, inclusive espécies cogenéricas, vivam no mesmo hospedeiro sem que ocorra uma competição aparente. Atyeo \& Windingstadt (1979) registraram quatro diferentes espécies de ácaros de pena ocupando diferentes regiões de uma única pena. Nas penas de $T$. albicollis da Ilha Grande, foram encontrados ácaros de pena em estádios adultos e também em estádios de ninfas. No entanto, a metodologia utilizada não permitiu identificar em qual estádio se encontravam os ácaros de penas e o microhábitat ocupado por ocasião da coleta no campo, não sendo possível determinar se os diferentes estádios de $P$. turdinus ocupavam partes diferentes de uma mesma pena.

Alguns gêneros de ácaros de pena são restritos a uma família ou mesmo a um único gênero de aves, enquanto outros não são tão restritos e têm sido registrados em um grande número de hospedeiros (GAud \& Atyeo 1996). Radford (1958) encontrou diferentes famílias de aves como Trochilidae, Parulidae e Icteridae, além da família Muscicapidae, parasitadas com Pterodectes. PARK \& Atyeo (1971) mostraram que Pterodectes estão associados principalmente com aves da ordem Passeriformes, sendo algumas espécies deste gênero restritas aos Apodiformes.

Os ácaros de pena apresentaram uma abundância mensal maior nos meses mais secos. $\mathrm{O}$ fato de T. albicollis apresentar uma abundância mensal de ácaros de pena menor nos meses mais chuvosos pode estar relacionado ao fato de no final do período chuvoso ocorrer a muda. Justamente os meses com menor abundância mensal de ácaros de pena (janeiro e fevereiro), foi o período em que foi registrada a ocorrência de muda em T. albicollis na Ilha Grande (obs.pessoal). Porém, MARINI et al. (1996) estudando a comunidade de aves do Estado do Paraná, observaram que a prevalência de ácaros de pena não foi homogênea entre os quatro anos de estudo, estações do ano e florestas com diferentes altitudes, mostrando assim que ocorre uma complexa dinâmica espacial e temporal dos ectoparasitos e seus hospedeiros. Portanto, outros fatores além do ciclo de muda podem estar atuando para explicar a maior abundância mensal dos ácaros nos meses mais secos para T. albicollis na Ilha Grande. Para uma melhor compreensão dos fatores relativos à dinâmica das relações parasito e hospedeiro são necessários mais estudos sobre este tema.

Os resultados do presente estudo indicam que, com os métodos utilizados, os ácaros de pena e os carrapatos não influenciaram negativamente T. albicollis, particularmente quanto à massa corporal. Isto sugere não ocorrer uma relação parasitohospedeiro dos ácaros de pena e dos carrapatos com T. albicollis na área estudada.

Revista Brasileira de Zoologia 22 (2): 419-423, junho 2005

\section{AGRADECIMENTOS}

Este estudo é parte dos resultados do Programa de Ecologia, Conservação e Manejo de Ecossitemas do Sudeste Brasileiro, Departamento de Ecologia da Universidade do Estado do Rio de Janeiro (UERJ). Os autores agradecem aos funcionários do Centro de Estudos Ambientais e Desenvolvimento Sustentável - CEADS no Campus Regional da UERJ na Ilha Grande, à Sub-Reitoria de Pós-Graduação e Pesquisa SR-2, ao Dr. João Luiz H. Faccini, do Departamento de Parasitologia da Universidade Federal Rural do Rio de Janeiro pela ajuda na montagem das lâminas e na identificação preliminar dos ácaros de pena; a Dra. Marinete Amorim pela revisão do artigo, ao Dr. Miguel A. Marini pela ajuda com as referências bibliográficas, aos colegas do Laboratório de Ecologia de Aves da UERJ: E.M.Almeida, R.V.Marques, M.B.Vecchi e P.D.Ritter pela ajuda no trabalho de campo e à Gerência de Meio Ambiente da Eletrobrás Termonuclear S.A./Eletronuclear, pelos dados de normal fornecidos. Ao Conselho Nacional de Desenvolvimento Científico e Tecnológico - CNPq pela concessão de bolsa de mestrado à primeira autora (Processo $\left.\mathrm{n}^{\circ} 141952 / 00-8\right)$ e de Produtividade em Pesquisa para a segunda autora (Processo n ${ }^{\circ}$ 301524/88-2).

\section{REFERÊNCIAS BIBLIOGRÁFICAS}

Alves, M.A.S. 1997. Effects of ectoparasites on the Sand Martin Riparia riparia nestlings. IBIS, Londres, 139: 494-496.

Amorim, M. \& N.M. Serra-Freire. 1999. Chave dicotômica para identificação de larvas de algumas espécies do gênero Amblyomma Koch, 1844 (Acari: Ixodidae). Entomología y Vectores, Rio de Janeiro, 6 (1): 75-90.

Atyeo, W.T. \& R.M. WindingSTAD. 1979. Feather mites of the grater sandhill crane. Journal of Parasitology, Cambridge, 65: 650658.

Begon, M.; J.L. Harper \& C.R. Towsend. 1990. Ecology - Individuals, populations and communities. London, Blackwell Scientific Publications, $2^{\text {nd }}$ ed., 945p.

Blanco, G. \& O. Frías. 2001. Symbiotic feather mites synchronize dispersal and population growth with host sociality and migratory disposition. Ecography, Copenhagen, 24: 113-120.

Blanco,G.; J.L. Tella \& J. Potti. 2001. Feather mites on birds: costs of parasitism or conditional outcomes? Journal of Avian Biology, Copenhagen, 32: 271-274.

Brown, C.R. \& M.D. Brown. 1986. Ectoparasitism as a cost of coloniality in cliff swallows (Hirundo pyrrhonota). Ecology, Washington, 67: 1206-1218.

Bush, A.O.; K.D. Lafferty; J.M. Lotz \& A.W. Shostak. 1997. Parasitology meets ecology on its own terms: Margolis et al. Revisited. Journal of Parasitology, Cambridge, 83: 575-583.

Снов, J. \& K.C. Кім. 1987. Community structure of arthropod ectoparasites on Alaskan seabirds. Canadian Journal of Zoology, Ottawa, 65: 2998-3005.

Davidson, W.R.; D.A. Siefken \& L.H.Creekmore. 1994. Seasonal and annual abundance of Amblyomma americanum (Acari: 
Ixodidea) in Central Georgia. Jounal of Medical Entomology, Lanham, 31 (1): 67-71.

Faccini, J.L.H.; J. Gaud. \& W.T. Atyeo. 1976. Descrição de Eurydiscalges g.n. (Analgidae: Sarcoptiformes) parasitos de Psittacidae da América do Sul. Revista Brasileira de Biologia, São Paulo, 36 (3): 701-707.

GAud, J. 1961. Six generes nouveaux de Sarcoptiformes plumicoles (Analgesoidea). Acarologia, Paris, 3: 78-95.

Gaud, J. \& W.T. Aтүeo. 1996. Feather mites of the world (Acarina, Astigmata): the supraspecific taxa. Part I. Annales Musée Royal de L'Afrique Centrale, Sciences Zoologiques, Tervuren, 277: 1-187.

HaRper, D.C.G. 1999. Feather mites, pectoral muscle condition, wing lenght and plumage colorationof passerines. Animal Behaviour, London, 58: 553-562.

KeIRANs, J.E.. \& L.A. Durden. 1998. Illustrated key to nymphs of the tick genus Amblyomma (Acari: Ixodidae) found in the United States. Journal of Medical Entomology, Lanham, 35 (4): 489-495.

Krantz, G.W. 1971. Manual of Acarology. Oregon, Oregon State University Press, $3^{\text {rd }}$ ed., 335p.

Lehman, T. 1993. Ectoparasites direct impact on host fitness. Parasitology Today, Amsterdam, 9: 8-13.

Linardi, M.P.; J.R. Botelho \& H.C. Cunha. 1985. Ectoparasitos de roedores da região urbana de Belo Horizonte. MG III. Índices pulicilianos, anoplurianos e acarinos em Rattus norvegicus norvegicus. Memórias do Instituto Oswaldo Cruz, Rio de Janeiro 80: 277-284.

Lope, F. \& A.P. Moller. 1993. Effects of ectoparasites on reproduction of their swallow hosts: a cost of being multi-brooded. Oikos, Copenhagen, 67: 557-562.

Lopes, C.; R. Leite.; M. Labruna; P. Oliveira.; L. Borges; Z. Rodriguez.; H.Carvalho; C. Freitas. \& C. Júnior. 1998. Host Specificity of Amblyomma cajennense (Fabricius, 1787) (Acari: Ixodidae) with comments on the drop off rythm. Memórias do Instituto Oswaldo Cruz, Rio de Janeiro, 93: 347-351.

Loye, J. \& S. Carrol. 1995. Birds, bugs and blood: avian parasitism and conservation. Trends in Ecology and Evolution, Amsterdam, 10 (6): 232-235.

Marini, M.A.; B.L.Reinert; M.R. Bornschein; J.C. Pinto \& M.A. Pichorim. 1996. Ecological correlates of ectoparasitism on Atlantic forest birds, Brazil. Ararajuba, Belo Horizonte, 4 (2): 93-102.

Merino, S. \& J. PotTr. 1995. Mites and blowflies decrease growth and survival in nestling Pied Flycatchers. Oikos, Copenhagen, 73: 95-103.

MolLer, A.P. 1990. Effects of parasitism by a haematophagous mite on reproduction in the Barn Swallow. Ecology, Washington, 71: 2345-2357.

Moss, W.W. \& J.H. CAmiN. 1970. Nests parasitism, productivity and clutch size in swallows. Functional Ecology, Oxford, 5: 351-359

Mumcuoglu, Y. \& R. Muller. 1974. Parasitsche Milben und Wurmer als Todesurache eineskhus Bubo bubo. Der Ornithologishe Beobacher, Alemanha, 71: 289-294.

Neves, R.L.; A.M.I.Farias; W.R. Telino JúnIor; M.ArzuA; M.C.N. BoteLHo \& M.C.A. Lima. 2000. Ectoparasitismo em aves silvestres (Passeriformes: Emberizidae) de Mata Atlântica, Igarassu, Pernambuco. Melopsittacus, Belo Horizonte, 3 (2): 64-71.

Park, C.H. \& W.T. Atyeo. 1971. A Generic Revision of the Pterodectinae, a new subfamily of feather mites (Sarcoptiformes: Analgoidea). Bulletin of The University of Nebraska State Museum, Nebraska, 9 (3): 47-49.

Perez, T.M. \& W.T. Atyeo. 1984. Site selection of the feather and quill mites of Mexican Parrots, p: 563-570. In: D.A. GRIFFITHS \& C.E. Bowman (Eds). Acarology VI. Chichester, Ellis Horwood, vol. 1, 1296p.

Philips, J.R. 1990. What's bugging your birds? Avian parasitic arthropods, p. 155-203. In: D.R. LuDwIG (Ed.). Wildlife Rehabilitation. Minnesota, Burgess Publishing, vol. 8.

RADFORD, C.D. 1958. The host-parasite relationships of the feather mites. Revista Brasileira de Entomologia, São Paulo, 8: 107-170.

Rojas, R.; M.A. Marini; M.T.Z. Coutinho. 1999. Wild Birds as hosts of Amblyomma cajennense (Fabricius, 1787) (Acari: Ixodidae). Memórias do Instituto Oswaldo Cruz, Rio de Janeiro, 94 (3): 315-322.

SCHALL, J.J. 1983. Lizard malaria: cost vertebrate host's reproductive success. Parasitology, Cambridge, 87: 1-6.

Sorci, G.; J. Clobert; Y. Michalakis. 1996. Cost of reproduction and cost of parasitism in the common lizard Lacerta vivipara. Oikos, Copenhagen, 76: 121-130.

Thompson, C.W.; N. Hillgarth; M. Leu \& H.E. McClure. 1997. High parasite load in house finches (Carpodacus mexicanus) is correlated with reduced expression of a sexually selected trait. American Naturalist, Chicago, 149: 270-294.

ZAR, J.H. 1984. Biostatistical analysis. New Jersey, PrenticeHall, Engewood Cliffs, $2^{\text {nd }}$ ed., 718p.

Recebido em 05.VIII.2004; aceito em 24.V.2005. 